\title{
Prophylactic antibiotic bundle compliance and surgical site infections: an artificial neural network analysis
}

\author{
Steven Walczak', Marbelly Davila ${ }^{2,3}$ and Vic Velanovich ${ }^{4 *}$ (D)
}

\begin{abstract}
Background: Best practice "bundles" have been developed to lower the occurrence rate of surgical site infections (SSI's). We developed artificial neural network (ANN) models to predict SSI occurrence based on prophylactic antibiotic compliance.

Methods: Using the American College of Surgeons National Quality Improvement Program (ACS-NSQIP) Tampa General Hospital patient dataset for a six-month period, 780 surgical procedures were reviewed for compliance with SSI guidelines for antibiotic type and timing. SSI rates were determined for patients in the compliant and noncompliant groups. ANN training and validation models were developed to include the variables of age, sex, steroid use, bleeding disorders, transfusion, white blood cell count, hematocrit level, platelet count, wound class, ASA class, and surgical antimicrobial prophylaxis (SAP) bundle compliance.
\end{abstract}

Results: Overall compliance to recommended antibiotic type and timing was $92.0 \%$. Antibiotic bundle compliance had a lower incidence of SSI's (3.3\%) compared to the non-compliant group $(8.1 \%, p=0.07)$. ANN models predicted SSI with a 69-90\% sensitivity and 50-60\% specificity. The model was more sensitive when bundle compliance was not used in the model, but more specific when it was. Preoperative white blood cell (WBC) count had the most influence on the model.

Conclusions: SAP bundle compliance was associated with a lower incidence of SSI's. In an ANN model, inclusion of the SAP bundle compliance reduced sensitivity, but increased specificity of the prediction model. Preoperative WBC count had the most influence on the model.

Keywords: Surgical site infection, Prophylactic antibiotic bundle compliance, Artificial neural networks

\section{Background}

Surgical site infections (SSI's) are a common problem after many types of operations. Approximately $2 \%$ of all patients who undergo an operation in the United States will develop an SSI [1, 2], with some types of operations, such as colonic surgery, being much higher [3]. In addition to the suffering this causes patients, it is a substantial financial burden [4]. This has led to efforts to identify risk factors for SSI's and to reduce the incidence of SSI.

There are numerous risk factors for the development of SSI's. The July, 2017 American College of Surgeons National Quality Improvement Program (ACS-NSQIP) SSI

\footnotetext{
* Correspondence: vvelanov@health.usf.edu

${ }^{4}$ Division of General Surgery, University of South Florida, Tampa, FL, USA

Full list of author information is available at the end of the article
}

model identified 28 statistically significant risk factors [5]. These include male sex, age, race, type of operation, operation complexity, American Society of Anesthesiologists' (ASA) classification, obesity/underweight, smoking status, inpatient surgery, wound classification, preoperative sepsis, surgical specialty, steroid use, diabetes mellitus, sodium level, chronic obstructive pulmonary disease, alkaline phosphatase level, functional status, bleeding disorders, weight loss, ventilator dependence, albumin level, dyspnea, creatinine level, hypertension, and thrombocytosis. Although these are identified as individual risk factors, how they interact with each other to potentiate or mitigate the risk for an individual patient is not well known.

General approaches to reducing SSI's have been to improve the individual patient's risk profile or to apply 
specific practices to mitigate risk. For example, Alexander, et al. [6] recommended an aseptic operating room environment, preoperative antiseptic bathing, hair removal by clipping, skin decontamination, drapes, bacterial-resistant suture, prophylactic antibiotics, maintenance of normal body temperature, maintenance of optimal oxygen saturation, glucose control, minimizing blood transfusions, judicious fluid management and, when necessary, delayed primary closure [6]. The value of each of these recommendations, let alone their use in combination, is variable.

There have been numerous attempts to create protocols to reduce the incidence of SSI's. One of the earliest was the Surgical Care Improvement Program (SCIP), which put forth guidelines aimed at SSI reduction [7]. However, the practical results of SCIP implementation have been mixed at best [8].
This has led others to develop SSI reduction strategies based on evidence-based best practices. Some of these SSI reduction "bundles" have had more success [9], especially in more high-risk operations such as colorectal surgery $[10,11]$.

The American College of Surgeons National Surgical Quality Improvement Program (ACS-NSQIP) allows hospitals to obtain risk-adjusted data of their outcomes, including SSI. The purpose is to provide hospitals with reliable data that they can use to implement quality improvement initiatives. ACS-NSQIP provides best practice guidelines to help with these quality improvement initiatives, including one for SSI (Table 1) [12]. Nevertheless, because the occurrence of SSI is complex with only a few factors in the surgeon's control, we hypothesized that there was more to this phenomenon.

Table 1 ACS-NSQIP Best Practice SSI Prevention Bundle

\begin{tabular}{|c|c|c|}
\hline Preoperative & Intraoperative & Postoperative \\
\hline Patient-Related & Patient-Related & Patient-Related \\
\hline $\begin{array}{l}\text { Encourage patient to discontinue tobacco } \\
\text { use for at least } 30 \text { days prior to operation }\end{array}$ & $\begin{array}{l}\text { Monitor and maintain glucose levels } \\
(<200 \mathrm{mg} / \mathrm{dl}) \text { in cardiothoracic surgery } \\
\text { patients, including non-diabetic patients }\end{array}$ & $\begin{array}{l}\text { Monitor and maintain glucose levels } \\
(<200 \mathrm{mg} / \mathrm{dl}) \text { in cardiothoracic surgery } \\
\text { patients, including non-diabetic patients }\end{array}$ \\
\hline $\begin{array}{l}\text { Identify and treat all non-surgical site } \\
\text { infections prior to surgery. Postpone elective } \\
\text { operations if necessary }\end{array}$ & $\begin{array}{l}\text { Discontinue prophylactic antibiotics within } \\
24 \mathrm{~h} \text { after noncardiac surgery and } 48 \mathrm{~h} \text { after } \\
\text { cardiac surgery }\end{array}$ & $\begin{array}{l}\text { Discontinue prophylactic antibiotics within } \\
24 \mathrm{~h} \text { after noncardiac surgery and } 48 \mathrm{~h} \text { after } \\
\text { cardiac surgery }\end{array}$ \\
\hline $\begin{array}{l}\text { Administer prophylactic antibiotics within } \\
\text { one hour prior to surgery (vancomycin and }\end{array}$ & $\begin{array}{l}\text { Cover primarily closed incisions with a sterile } \\
\text { dressing for } 24 \text { to } 48 \mathrm{~h} \text { postoperatively. Wash }\end{array}$ & $\begin{array}{l}\text { Cover primarily closed incisions with a sterile } \\
\text { dressing for } 24 \text { to } 48 \mathrm{~h}\end{array}$ \\
\hline
\end{tabular}
fluoroquinolones should be administered two hours prior to surgery). Select the appropriate antimicrobial prophylaxis based on evidence-based guidelines

Adjust the dose of the prophylactic antibiotics for morbid obesity

Provider-Related

Keep nails short. Do not wear artificial nails or hand or arm jewelry

Clean underneath fingernails prior to first daily surgical scrub. Complete a two to five minute preoperative scrub using appropriate antiseptic or use alcohol-based surgical antiseptic hands before and after any contact with surgical site.

Provider-Related

Wear a cap or hood to fully cover head/facial hair and a surgical mask to cover nose/mouth when entering the operating room (if operation is about to begin, is underway, or surgical instruments are exposed) and until the conclusion of the operation.

Use surgical gown and drapes that are liquid resistant

\author{
Wear sterile gloves if a scrubbed surgical team \\ member \\ Change surgical scrubs if grossly soiled or \\ contaminate \\ System-Related \\ Comply with standards regarding operating \\ room asepsis \\ When visible contamination of surfaces/ \\ equipment with body fluids occurs, use an \\ Environmental Protection Agency-approved \\ cleaning solution to clean affected area before \\ next operation
}


Artificial neural networks (ANNs) are a nonparametric machine learning method based on modeling the neuronal activity of the human brain. Neuronal activity is simulated using processing elements referred to as neurodes that are arranged in layers and connected to neurodes in subsequent layers through a connection which carries a weight value. The weighted values of the connections indicate the strength of the neuronal signal from one neurode to the next. ANNs can develop predictive models based on the program's ability to "learn" through adjustment of the weight values on the interlayer connections. ANN learning enables the ANN to accurately model nonlinear relationships between factors related to a clinical problem and the clinical problem's results. We chose ANN as the machine learning method explicitly because of its ability to identified "hidden" relationships in data sets that may not be obvious through standard, linear models.

We suspected that in addition to the surgical antimicrobial prophylaxis (SAP) bundle compliance, other factors may also drive SSI occurrences. Our premise is that the occurrence of a SSI is a complex process, due to the interaction of numerous patient risk factors and patient-care process factors that occur with each operation. Our aim is to apply ANNs to SSI occurrence across surgical specialties to determine the efficacy of ANN models to predict SSI occurrence and to assess the influence of ACS-NSQIP compliance in predicting SSI occurrence. Our hypothesis is that SAP bundle compliance will reduce the incidence of SSI occurrences and be predictive of an SSI-free surgical outcome.

\section{Methods}

This study was approved by the institutional review boards of the University of South Florida and Tampa General Hospital.

All patients who underwent operations at Tampa General Hospital, a large, urban, tertiary, teaching hospital located in Tampa, Florida, USA, and were subsequently submitted to the ACS-NSQIP data base and reported back to Tampa General Hospital from 1 July 2015 to 31 December 2015 were included in this study. The primary outcome measure was SSI occurrence, and the secondary outcome measure was SAP compliance. Patients who underwent operations and whose data were not submitted to the ACS-NSQIP data base or were outside this time frame were excluded. Use of the ACS-NSQIP definitions for patient risk factors and SSI occurrence was done to have uniform definitions of risk variables and SSI's. A total of 780 distinct records were obtained for analysis. Once this list was generated, variables including age, sex, operation, surgeon, surgical specialty and wound classification were collected for SSI occurrence analysis. These variables were chosen because they could be consistently found in the records of all patients and would provide a profile of the breath of operative experience at Tampa General Hospital. Specifically, for surgeon and surgical specialty, we wished to identify if individual surgeons or surgical specialty, as they generally adhered to similar practice patterns, influence SSI occurrences. The records of these patients were reviewed for adherence to the ACS-NSQIP best practice SSI prevention bundle [12]. Table 1 presents the entire ACS-NSQIP recommend SSI reduction bundle. We specifically determined whether two aspects of the bundle were adhered to: the type of antibiotic and timing of administration. Other aspects of the bundle could not be assessed due to lack of pertinent information in the record. These data would provide the basis of the incidence of SSI occurrences in patients compliant and non-compliant to the SAP bundle.

\section{Statistical analysis}

We compared the group of patients where both the type of antibiotic and the timing of the antibiotic (SAP bundle) were compliant with the ACS-NSQIP SSI reduction recommendations to the group where they were not compliant. This was the basic determination if compliance was associated with lower SSI rates. Variability of antibiotic timing was also determined. Categorical data were described as totals, frequencies, and percentages; continuous variables were described as means with standard deviation and medians with interquartile ranges. Univariate comparisons were assessed for covariates and outcome variable between SAP compliant and SAP noncompliant applying the chi-square test or Fisher's exact test for categorical variables. A $p$-value of 0.05 or less was considered statistically significant. Data were analyzed with SAS 9.4 software, SAS Institute Inc., Cary, NC, USA. For this portion of the analysis, all 780 patient records were used.

\section{Artificial neural network analysis}

ANNs have been shown to be universal approximators for arbitrarily complex, including nonlinear, problems [13], and thus ANN models were developed to predict SSI occurrence. The 11 independent variables analyzed were: age (in years), sex (male/female), steroid use (present/not present), bleeding disorders (present/not present), transfusion (present/not present), white blood cell count (WBC/ $\mathrm{mm}^{3}$ ), hematocrit level (\%), platelet count (platelets $/ \mathrm{mm}^{3}$ ), wound classification (class 1, 2, 3, or 4), ASA class (class 1, $2,3,4$, or 5), and NSQIP surgical antimicrobial prophylaxis bundle compliance (present/not present). The dependent variable was the occurrence of an SSI, which is represented with a value 1 for an SSI and a value -1 for no SSI. As ANN can only analyze complete data sets, surgical population sample data with missing values were eliminated from the ANN training and evaluation data. A 
total of 646 clean records were used for training and validating the ANNs.

The training algorithm used for the ANN models was backpropagation, which is the most commonly used training algorithm in medical ANN models [14, 15]. Various architectures were designed following best practices [16, 17] using both single hidden layer and two hidden layer designs, with various quantities of neurodes per hidden layer. For each architecture for each of the ANN models, 3-fold cross validation was used to separate training and validation data samples. The two hidden layer architectures performed the best and are the ones reported below.

Two distinct ANN models were developed using the NeuralWare ${ }^{\curvearrowleft}$ NeuralWorks Professional II Plus $\odot$ neural network shell tool. The first ANN model included the NSQIP SAP bundle compliance variable and the second ANN model used the other 10 variables without the NSQIP SAP bundle compliance variable. The optimal architecture for the full 11 variables ANN model is presented in Fig. 1 (the other ANN architectures are similar). The purpose of the distinct ANN models is to evaluate the importance and influence of specific variables using the leave-one-variableout method, which is a commonly utilized technique for determining variable influence in ANNs [18, 19]. One additional model was developed that also left out the sex variable, in addition to the bundle compliance variable, to evaluate its influence on SSI predictions.

\section{Determination of relative importance}

After all the different ANN architectures and models were evaluated, one further ANN model that duplicated the complete 11 variable set was developed using the JustNN@ neural network shell tool to further evaluate variable contributions to SSI prediction. JustNN@ tries to determine variable influence using the sum of the weighted connections between processing elements in the ANN [20].

\section{Results}

A total of 780 surgical procedures from 9 surgical specialties including 112 surgeons and 220 distinct CPT coded operations were collected. Table 2 presents the patient demographic information and SSI outcome stratified by SAP bundle compliance status. Over $60 \%$ of the patients were female, and the range of ages was from 19 to 94 years old. The distribution of wound classification was clean $55.0 \%$, clean-contaminated $37.8 \%$, contaminated $2.8 \%$, and dirty $4.4 \%$. The distribution of surgical specialties was general surgery $40.4 \%$, gynecology $16.3 \%$, neurosurgery $12.1 \%$, orthopedic surgery $11.9 \%$, urology $8.6 \%$, vascular surgery $5.6 \%$, plastic surgery $1.9 \%$, thoracic surgery $1.9 \%$ and otolaryngology $1.2 \%$. Individual surgeon data was not analyzed as there were too few operations for each surgeon to make for a meaningful analysis. The overall compliance of the prophylactic antibiotic SAP bundle was $92.0 \%$ (718 of 780 patients), with appropriate antibiotic compliance of $94.6 \%$ (738 of 780 patients) and appropriate timing of 92.1\% (719 of 780 patients). Of note, patients in the non-compliant group were more frequently smokers and underwent emergent operations, while patients in the compliant group were more frequently undergoing elective operations (Table 2). There were no other statistically significant differences between the groups. Table 3 shows the mean time of

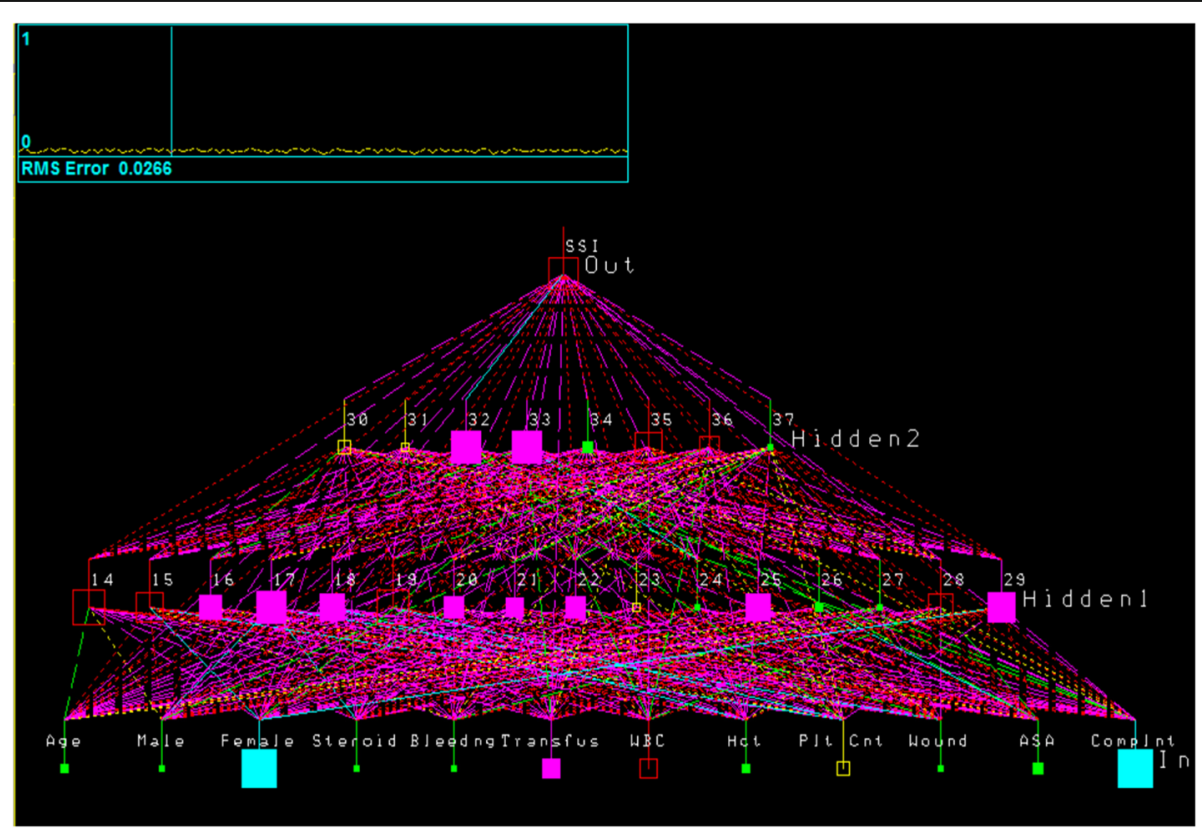

Fig. $1 \mathrm{SSI}$ Prediction Artificial Neural Network Architecture (w/ bundle compliance) 
Table 2 Characteristics of the Study Cohort Stratified by Surgical Antimicrobial Prophylaxis (SAP) Bundle

\begin{tabular}{|c|c|c|c|c|}
\hline Characteristics & Total No. Surgical Procedures (\%) $n=780$ & No. SAP Bundle Compliant $n=718$ & No. SAP Bundle Noncompliant $n=62$ & $p$-value* \\
\hline \multicolumn{5}{|l|}{ Sex } \\
\hline Male & 307 (39.4) & $280(39)$ & $27(43.5)$ & \\
\hline Female & $473(60.6)$ & $438(61)$ & $35(56.5)$ & 0.48 \\
\hline Age, y & & & & 0.07 \\
\hline$<65$ & $547(70.1)$ & $498(69.4)$ & $49(79)$ & \\
\hline$\geq 65$ & $233(29.9)$ & $220(30.6)$ & $13(21)$ & \\
\hline BMl & & & & 0.77 \\
\hline$<30$ & $414(53.1)$ & $380(52.9)$ & $34(54.8)$ & \\
\hline$>=30$ & $366(46.9)$ & $338(47.1)$ & $28(45.2)$ & \\
\hline ASA score & & & & 0.92 \\
\hline$<3$ & $297(38.1)$ & $273(38)$ & $24(38.7)$ & \\
\hline$\geq 3$ & $483(61.9)$ & $445(62)$ & $4.8(61.3)$ & \\
\hline Diabetes & & & & 0.44 \\
\hline Yes & $128(16.4)$ & $120(16.7)$ & $8(12.9)$ & \\
\hline No & 652 (83.6) & $598(83.3)$ & $54(87.1)$ & \\
\hline COPD & & & & 0.92 \\
\hline Yes & $27(3.5)$ & $25(3.5)$ & $2(3.2)$ & \\
\hline No & 753 (96.5) & $693(96.5)$ & $60(96.8)$ & \\
\hline Smoker & & & & 0.001 \\
\hline Yes & $123(15.8)$ & $104(14.5)$ & 19 (30.6) & \\
\hline No & $657(84.2)$ & $614(85.5)$ & $43(69.4)$ & \\
\hline On steroids & & & & 0.15 \\
\hline Yes & $44(5.6)$ & $38(5.3)$ & $6(9.7)$ & \\
\hline No & 736 (94.4) & $680(94.7)$ & $56(90.3)$ & \\
\hline Emergent case & & & & $<0.001$ \\
\hline Yes & $38(4.9)$ & $26(3.6)$ & $12(19.4)$ & \\
\hline No & $742(95.1)$ & $692(96.4)$ & $50(80.6)$ & \\
\hline Elective case & & & & $<0.001$ \\
\hline Yes & $624(80)$ & $594(82.7)$ & $30(48.4)$ & \\
\hline No & $156(20)$ & $124(17.3)$ & 32 (51.6) & \\
\hline SSI & & & & 0.07 \\
\hline Yes & $29(3.7)$ & $24(3.3)$ & $5(8.1)$ & \\
\hline No & 751 (96.3) & $694(96.7)$ & 57 (91.9) & \\
\hline
\end{tabular}

* $p$-value refers to comparison between SAP compliant and SAP noncompliant and are significant at alpha level of 0.05 . SSI indicates surgical site infection; ASA, American Society of Anesthesiologists; BMI, body mass index

antibiotic administration to surgical incision based on specialty with the 25 th, 50 th percentile and 75 th percentile times of the time distribution. The median time to administration was within $7 \mathrm{~min}$ among the specialties. Three specialties had fourth quartile times of greater than $30 \mathrm{~min}$, while one had fourth quartile time of less than $20 \mathrm{~min}$. The distribution of SSI's types was superficial 31\% (9 of 29 patients), deep 38\% (11 of 29 patients) and organ space 31\% (9 of 29 patients). Overall, cases in which there was compliance with both antibiotic type and timing had an SSI rate of $3.3 \%$ (24 of 718 patients), while those which were noncompliant had a rate of $8.1 \%$ (5 of 62) with a $p=0.07$.

The constructed neural network is visualized in Fig. 1. The lines represent both positive and negative interactions between the neurodes. There were two hidden layers identified. The first layer had 16 neurodes, while the second layer had eight. It should be noted that there are multiple positive and negative interactions between the neurodes, implying that the occurrence of an SSI is a complex process. The Professional II Plus@ ANN models' results are 
Table 3 Time from Surgical Antimicrobial Prophylaxis (SAP) Administration to Incision (minutes)

\begin{tabular}{|c|c|c|c|c|c|c|}
\hline Surgical Specialty, No. Surgical Procedures (\%) & Mean & Median & p25th & p75th & IQR & SD \\
\hline General Surgery, 315 (50.4) & 28.6 & 13.0 & 8.0 & 24.0 & 16.0 & 90.0 \\
\hline Gynecology, 127 (16.3) & 17.3 & 17.0 & 11.0 & 22.0 & 11.0 & 9.8 \\
\hline Neurosurgery, 95 (12.2) & 36.1 & 18.0 & 11.0 & 33.8 & 22.8 & 103.7 \\
\hline Orthopedics, 93 (11.9) & 41.1 & 20.0 & 14.0 & 32.5 & 18.5 & 142.3 \\
\hline Urology, 67 (8.6) & 19.2 & 16.0 & 8.5 & 27.0 & 18.5 & 13.0 \\
\hline Vascular, 44 (5.6) & 22.7 & 19.0 & 10.5 & 28.0 & 17.5 & 18.7 \\
\hline Plastics, 15 (1.9) & 15.3 & 18.0 & 6.0 & 22.5 & 16.5 & 8.6 \\
\hline Thoracic, 15 (1.9) & 37.5 & 14.0 & 7.5 & 36.0 & 28.5 & 63.8 \\
\hline ENT, 9 (1.2) & 15.7 & 16.0 & 11.0 & 20.5 & 9.5 & 5.1 \\
\hline
\end{tabular}

$\mathrm{P} 25^{\text {th }}=25^{\text {th }}$ percentile; $\mathrm{p} 75^{\text {th }}=75^{\text {th }}$ percentile; IQR $=$ interquartile range; $\mathrm{SD}=$ standard deviation

presented in Table 3. Overall the ANNs predicted SSI with a $69-90 \%$ sensitivity and a $60-50 \%$ specificity, depending on the variable set used to develop the corresponding ANN model. While use of the bundle compliance variable in the ANN model lead to a higher specificity (i.e., improves predictions of non-SSI occurrence), it leads to lower sensitivity (i.e., reduced the prediction of an SSI occurrence) (Table 4). The sex variable did not affect specificity (i.e., non-SSI predictions), but did reduce the sensitivity for SSI predictions by $10 \%$, though this was still $10 \%$ greater than the sensitivity of the model that included the ACS-NSQIP compliance variable.

The relative variable influence by summed weights, using the JustNN@ shell tool, is presented in Fig. 2, with higher values indicating greater significance. The sum of connection weights analysis supports the leave-onevariable-out analysis and indicates that bundle compliance is not a significant variable for predicting SSI occurrence. The most important variable was preoperative WBC count with a value of 82 , while the least important one with any value was sex with a value of 6 . Bundle compliance was the fourth least important with a value of 13. Figure 2 also indicates that the presence of bleeding disorders and transfusion had no influence whatsoever with values of 0 .

\section{Discussion}

What this study demonstrates is that there are complex interactions among the patient factors (such as comorbidities, medication usage, and physiology as measured by laboratory values) the physician antibiotic ordering practices (such as which antibiotics are ordered), and system factors (such as timing of antibiotic administration) which can affect prediction of an SSI in an individual patient. The complexity of the interaction can clearly be seen in Fig. 1, in which the ANN identified two hidden layers of interactions with a total of 24 neurodes of both positive and negative interactions (Fig. 1). Specifically, the ANN models showed that compliance with a bundle of the appropriate antibiotic type and timing did not show a better sensitivity, although did show a better specificity, in the prediction of the development an SSI compared to the models that did not use this variable. We would like to emphasize that this study is not intended to determine if antibiotic bundle compliance reduces the incidence of SSI occurrences, but rather it has little value in predicting which patient will developed an SSI.

This study has demonstrated that when applied in a broad population of surgical patients and operations, the machine learning nonparametric method of ANNs using supervised backpropagation learning can predict SSI's with 69 to $90 \%$ sensitivity and 60 to $50 \%$ specificity, depending on the variable set chosen. The output of the ANN is a prediction for an individual patient about whether they will suffer a SSI following surgery. Higher sensitivity is desirable since this would enable focused monitoring and quicker intervention for patients more likely to suffer an SSI. Nevertheless, the question remains as to why inclusion of the SAP variable decreases the sensitivity of the ANN prediction model by 10 to 20 percentage points, yet improve specificity by about 10

Table 4 Results for ANN Models Predicting Occurrence of SSI

\begin{tabular}{lll}
\hline $\begin{array}{l}\text { ANN Model } \\
\text { (variables used) }\end{array}$ & $\begin{array}{l}\text { SSI prediction sensitivity } \\
(n=29)\end{array}$ & $\begin{array}{l}\text { No SSI prediction specificity } \\
(n=617)\end{array}$ \\
\hline All variables including NSQIP compliance variable & $69.0 \%$ & $60.1 \%$ \\
All variables excluding NSQIP compliance variable & $89.7 \%$ & $50.2 \%$ \\
All variables excluding NSQIP compliance and Sex variables & $79.3 \%$ & $50.2 \%$ \\
\hline
\end{tabular}




\section{Relative Variable Importance}

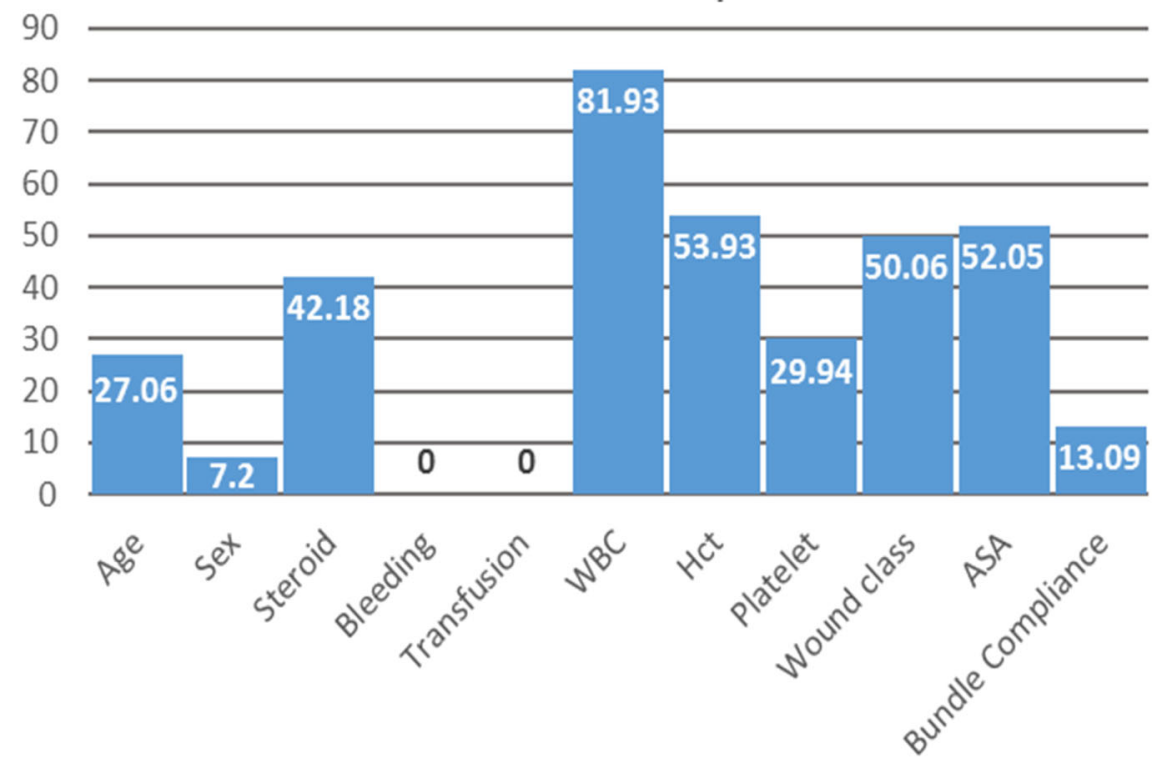

Fig. 2 Variable Influence for the ANN Model Using Sum of Connection Weights

percentage points? We do not have a good answer for this question, but speculate that there is some type of interaction with the previously mentioned patient factors. This will be an area of additional research.

Using a leave-one-variable-out methodology, the two variables NSQIP bundle compliance and sex were evaluated for their contribution to the SSI prediction models. The NSQIP bundle compliance variable was found to not contribute significantly to sensitivity for predicting SSI with a raw $20 \%$ and net $30 \%$ improvement in sensitivity when this variable was not present, though higher specificity was achieved when the bundle compliance variable was present. A different analysis using a sum of the connection weights methodology was also utilized and confirmed the lack of influence on predicting an SSI (regarding sensitivity) for both NSQIP bundle compliance and sex. Additionally, the sum of the connection weights analysis also indicated that the variables of bleeding disorders and transfusions had no effect.

Several studies have assessed the effectiveness of SSI bundles in reducing SSI occurrences with mixed results. Hawn, et al. [8] and Anthony, et al. [21] report that compliance with an SSI bundle did not reduce SSI rates. Kim, et al. [22] in a systematic review and meta-analysis of laparoscopic cholecystectomy found prophylactic antibiotic reduced superficial SSI's but not deep SSI's in low-risk patients. On the other hand, several studies have found benefit in colorectal surgery [23-25], neurosurgery [26], hysterectomy [27], as well as a board national surgical database [9]. In fact, in our study, compliance with the antibiotic type and timing was associated with a lower incidence of postoperative SSI, although it did not quite reach statistical significance. Part of the inconsistency could be because these studies use different bundles [10]; however, part could also be due to the fact that none of their analyses include patient specific factors in the development of SSI's. Studies of efficacy of bundle compliance have generally focused bundles in the randomized trials or retrospective cohorts, without accounting for individual patient factors. These interactions may affect the predictive value of compliance adherence to SSI development.

There are been numerous studies attempting to develop predictive models for the occurrence of SSI's. These have included major abdominal surgery [28], spinal surgery [29], vascular surgery [30], ventral hernia [31], and orthopedic surgery [32], among others [33]. However, there has been problems translating these prediction models to other datasets. For example, Bergquist, et al. [34] documented that several colorectal surgery SSI predictive models did not perform as well as their original studies when applied to another institution's dataset. Yet, studies on SSI prediction have generally focused on patient factors without addressing clinical practice, such as the use of prophylactic antibiotic bundles. Therefore, as with studies assessing efficacy of SSI reduction bundles, SSI prediction models have not assessed fully the interaction of patient factors with actual patient care.

Because the ANN models output a real value, the classification of SSI occurrence was made for all outputs above a pre-specified cutoff value, usually zero. However, 
modifying the cutoff value enables adjustments in the sensitivity and specificity, but there is a tradeoff. Lowering the cutoff value below zero will improve sensitivity, but at the same time may decrease specificity. Of course, it is pointless to predict that all patients will have an SSI so that no differentiation is performed by the ANN classification. As an example, the third ANN model that did not have both a NSQIP compliance variable and the sex variable had an identical specificity, but the sensitivity was only $79 \%$ as opposed to $90 \%$ for the model only missing the NSQIP compliance variable. Adjusting the cutoff downwards to make the third ANN model's sensitivity identical to the ANN model missing only the NSQIP compliance variable resulted in a specificity of $24 \%$. The lower specificity indicates that a greater number of patients who would not suffer an SSI would still need to be monitored for an SSI since they were incorrectly classified as false positives.

ANN is a type of nonparametric machine learning. It searches for "hidden" interactions among the variables which may be associated with the occurrence of a specific event. Other types of nonparametric machine learning programs include support vector machines [35] and conditional inference trees [36]. What we have found in this study is that the most relevant factor was preoperative white blood cell count, while bundle compliance was the fourth least relevant. Others have used machine learning program to predict SSI's. Ke, et al. [37] used a machine learning algorithm based on temporal changes in wound characteristics to create a model predicting time to SSI development. Soguero-Ruiz, et al. [38] used laboratory testing data with Gaussian process regression, time warping and imputation methods into a support vector machine to predict SSI's. Interestingly, their analysis, like ours, also showed that platelet count, white blood cell count, hemoglobin level, among others, as relevant factors in their prediction model.

There are several limitations to this study. One limitation is that due to the retrospective nature of the study, not all the recommended NSQIP bundle were well or consistently documented and, therefore, could not be included in the model. It is unknown how including these other factors could have affected the predictive value of compliance with the bundle. Another limitation is that not all possible architectures of ANN were attempted. It is possible that a different architecture could have further improved the sensitivity or the specificity or both for any of the ANN models. Furthermore, ANN modeling has not been applied to other SSI datasets. It is unclear how whether ANN modeling will yield similar results to other datasets. Lastly, we also did not compare our ANN model to experienced practitioners SSI risk assessment for an individual patient. Perhaps such practitioners would be more accurate. Therefore, the results reported should be viewed as the minimum achievable by an ANN SSI prediction model.

Future research is needed to further explore the significance of the 9 variables other than bundle compliance and sex to using the leave-one-variable-out method to further determine their influence on the SSI prediction models. Additional variable may also be evaluated by flipping the approach and adding one in to an existing model and examining the impact on sensitivity and specificity.

\section{Conclusion}

In conclusion, this study has provided evidence for the efficacy of using ANN models to predict SSI. ANN models can achieve almost $90 \%$ sensitivity for predicting SSI for a patient. Evidence from the ANN models indicate that knowledge of compliance with SSI reduction protocols is not a factor in predicting likely SSI occurrence. This is not to say that bundle compliance did not reduce the incidence of SSI's, only that other relevant factors outweigh it as a predictive factor. More importantly, this study is one of the few studies evaluating patient-specific factors as well as a clinical practice factors. Understanding the interaction is a potentially important new frontier in clinical prediction research.

\section{Abbreviations \\ ACS-NSQIP: American College of Surgeons National Surgical Quality Improvement Program; ANN: Artificial neural networks; SAP: Surgical antimicrobial prophylaxis; SCIP: Surgical Care Improvement Program; SSI: Surgical site infection; WBC/ $\mathrm{mm}^{3}$ : White blood count per cubic millimeter}

\section{Authors' contributions}

Drs. Walczak, Davila and Velanovich have made substantial contributions to the conception, design of the work; the acquisition, analysis, and

interpretation of data; and have drafted the work and substantively revised it; and have approved the submitted version; and have agreed both to be personally accountable for the author's own contributions and to ensure that questions related to the accuracy or integrity of any part of the work, even ones in which the author was not personally involved, are appropriately investigated, resolved, and the resolution documented in the literature. All authors read and approved the final manuscript.

\section{Funding}

This study was not funded.

\section{Availability of data and materials}

The datasets used and/or analyzed during the current study are available from the corresponding author on reasonable request.

Ethics approval and consent to participate

This study was approved by the Institutional Review Boards of both the University of South Florida and Tampa General Hospital.

\section{Consent for publication}

Not applicable

Competing interests

None of the authors have a conflict of interest with respect to this study. 


\section{Author details}

'School of Information and Florida Center for Cybersecurity, University of South Florida, Tampa, FL, USA. ${ }^{2}$ College of Business, Information and Technology Management, University of Tampa, 5 Tampa General Circle, Suite 740, Tampa, FL 33606, USA. ${ }^{3}$ Tampa General Hospital, Tampa, FL, USA. ${ }^{4}$ Division of General Surgery, University of South Florida, Tampa, FL, USA.

Received: 20 August 2019 Accepted: 26 November 2019

Published online: 07 December 2019

\section{References}

1. Mu Y, Edwards JR, Horan TC, Berrios-Torres SI, Fridkin SK. Improving riskadjusted measures of surgical site infection for the national healthcare safety network. Infect Control Hosp Epidemiol. 2011;32:970-86.

2. Centers for Disease Control and Prevention. Surgical site infection (SSI) event. 2017:1-31.

3. Hubner M, Diana M, Zanetti G, Eisenring MC, Demartines N, Troillet N. Surgical site infections in colon surgery: the patient, the hospital and the surgeon. Arch Surg. 2011;146:1240-5.

4. Shepard J, Ward W, Milstone A, Carlson T, Frederick J, Hadhazy E, Perl T. Financial impact of surgical site infections on hospitals: the hospital management perspective. JAMA-Surg. 2013;148:907-14.

5. ACS-NSQIP Semi-Annual Report (SAR) Supplement: Models Report, Release July, 2017. American College of Surgeons, Chicago, 2017.

6. Alexander JW, Solomkin JS, Edwards MJ. Updated recommendations for control of surgical site infections. Ann Surg. 2011:253:1082-93.

7. Bratzler DW, Hunt DR. The surgical infection prevention and surgical care improvement projects: national initiatives to improve outcomes for patients having surgery. Clin Infect Dis. 2006:322-30.

8. Hawn MT, Vick CC, Richman J, Holman W, Deierhoi RJ, Graham LA, Henderson WG, Itani KMF. Surgical site infection prevention: time to move beyond the surgical care improvement program. Ann Surg. 2011;254:494501

9. Koek MBG, Hopmans TEM, Soetens LC, Wille JC, Geerlings SE, Vos MC, van Benthem BHB, de Greeff SC. Adhering to a national surgical care bundle reduces the risk of surgical site infections. PLoS One. 2017;12(9):e0184200.

10. Tanner J, Padley W, Assadian O, Leaper D, Kiernan M, Edmiston C. Do surgical care bundles reduce the risk of surgical site infections in patient undergoing colorectal surgery? A meta-analysis and systematic review. Surgery. 2015;158:66-77.

11. Zywot A, Lau CSM, Fletcher HS, Paul S. Bundles prevent surgical site infections after colorectal surgery: meta-analysis and systematic review. J Gastrointest Surg. 2017;21:1915-30.

12. Ingraham AM, Shiloach M, Dellinger EP, Esmaola NF. Prevention of surgical site infections. Chicago: ACS NSQIP; 2009.

13. Hornik K, Stinchcombe M, White H. Multilayer feedforward networks are universal approximators. Neural Netw. 1989;2(5):359-66.

14. Amato F, López A, Peña-Méndez EM, Vaňhara P, Hampl A, Havel J. Artificial neural networks in medical diagnosis. J Appl Biomed. 2013;11:47-58.

15. Walczak $S$, Velanovich $V$. Improving prognosis and reducing decision regret for pancreatic cancer treatment using artificial neural networks. Decis Support Syst. 2018;106:110-8.

16. Walczak S, Cerpa N. Heuristic principles for the design of artificial neural networks. Information Software Tech. 1999;41(2):107-17.

17. Zhang GP. Avoiding pitfalls in neural network research. IEEE Trans Syst Man Cybern Part C Appl Rev. 2007;37:3-16

18. Tu JV. Advantages and disadvantages of using artificial neural networks versus logistic regression for predicting medical outcomes. J Clin Epidemiol. 1996:49(11):1225-31.

19. Walczak S. Evaluating medical decision making heuristics and other business heuristics with neural networks. In intelligent decision making: an Al-based approach 2008 (pp. 259-287). Springer, Berlin. Heidelberg.

20. Augasta MG, Kathirvalavakumar T. Reverse engineering the neural networks for rule extraction in classification problems. Neural Process Lett. 2012;35(2): 131-50.

21. Anthony T, Murray BW, Sum-Ping JT, Lenkovsky F, Vornik VD, Parker BJ, McFarlin JE, Hartless K, Huerta S. Evaluating an evidence-based bundle for preventing surgical site infection. Arch Surg. 2011;146:263-9.

22. Kim SH, Yu HC, Yang JD, Ahn SW, Hwang HP. Role of prophylactic antibiotics in elective laparoscopic cholecystectomy: a systematic review and meta-analysis. Ann Hepatobiliary Pancreat Surg. 2018;22:231-47.
23. Hoang JC, Klipfel AA, Roth LA, Vress M, Schechter S, Shah N. Colon and rectal surgery surgical site infection reduction bundle: to improve is to change. Am J Surg. 2019;217:40-5.

24. Keenan JE, Speicher PJ, Thacker JKM, Walter M, Kuchibhaltla M, Mantyh CR. The preventive surgical site infection bundle in colorectal surgery. JAMA Surg. 2014;149:1045-52.

25. Waits SA, Fritze D, Banerjee M, Zhang W, Kubus J, Englesbe MJ, Campbell DA Jr, Hendren S. Developing an argument for bundled interventions to reduce surgical site infection in colorectal surgery. Surgery. 2014;155:602-6.

26. Alotaibi AF, Hulou MM, Vestal M, Alkholifi F, Asgarzadeh M, Cote DJ, Bi WL, Dunn IF, Mekary RA, Smith TR. The efficacay of antibacterial prophylaxis against the development of meningitis after craniotomy: a meta-analysis. World Neurosurg. 2016:90:597-603.

27. Ayeleke RO, Mourad S, Marjoribanks J, Calis KA, Jordan V. Antibiotic prophylaxis for elective hysterectomy. Cochrane Database Syst Rev. 2017;6: CD004637.

28. Ejaz A, Schmidt C, Johnston FM, et al. Risk factors and prediction model for inpatient surgical infection after major abdominal surgery. J Surg Res. 2017; 217:153-9.

29. Janssen DMC, van Kuijk SMJ, d'Aumerie BB, Willems PC. External validation of a prediction model for surgical site infection after thoracolumbar spine surgery in a Western European cohort. J Orthop Surg Res. 2018 May 16; 13(1):114

30. Bennett KM, Levinson H, Scarborough JE, Shortell CK. Validated prediction model for severe groin wound infection after lower extremity revascularization procedures. J Vasc Surg. 2016;63:414-9.

31. Liang MK, Goodenough CJ, Martindale RG, Roth JS, Kao LS. External validation of the ventral hernia risk score prediction of surgical site infections. Surg Infect. 2015;16:36-40.

32. Lewallen LW, Maradit Kremers H, Lahr BD, Mabry TM, Steckelberg JM, Berry DJ, Hanssen AD, Berbari EF, Osmon DR. External validation of the national healthcare safety network risk models for surgical site infections in total hip and knee replacements. Infect Control Hosp Epidemiol. 2014:35:1323-9.

33. Li X, Nylander W, Smith T, Hans S, Gunnar W. Risk factors and prediction model development of thirty-day postoperative surgical site infections in veterans administrative surgical population. Surg Infect. 2018;19:278-85.

34. Bergquist JR, Thiels CA, Etzioni PA, Habermann EB, Cima RR. Failure of colorectal surgical site infection predictive models applied to an independent dataset: do they add value or just confusion? J Am Coll Surg. 2016;222:421-38.

35. Syed Z, Rubinfeld IS, Patton JH Jr, Ritz J, Jordan J, Doud A, Velanovich V. Using procedural codes to supplement risk adjustment: a non-parametric learning approach. J Am Coll Surg. 2011;212:1086-93.

36. Hartney MM, Liu Y, Velanovich V, Fabri P, Marcet J, Greico M, Huang S, Zayas-Castro J. Bounceback branchpoints: using conditional inference tree to analyze readmissions. Surgery. 2014;156:842-8.

37. Ke C, Jin Y, Evans H, Lober B, Qian X, Liu J, Huang S. Prognostics of surgical site infections using dynamic health data. J Biomed Inform. 2017;65:22-33.

38. Soguero-Ruiz C, Wang F, Jenssen R, Augestad KM, Rojo-Alvarez J-L, MoraJimenez I, Lindsetmo R-O, Skrovseth SO. Data-driven temporal prediction of surgical site infection. AMIA Symp Proc. 2015;2015:1164-73.

\section{Publisher's Note}

Springer Nature remains neutral with regard to jurisdictional claims in published maps and institutional affiliations.

Ready to submit your research? Choose BMC and benefit from:

- fast, convenient online submission

- thorough peer review by experienced researchers in your field

- rapid publication on acceptance

- support for research data, including large and complex data types

- gold Open Access which fosters wider collaboration and increased citations

- maximum visibility for your research: over $100 \mathrm{M}$ website views per year

At $\mathrm{BMC}$, research is always in progress.

Learn more biomedcentral.com/submissions 